\title{
Nonfouling Surface Coatings Based on Poly(2-methyl-2-oxazoline)
}

\author{
Bidhari Pidhatika ${ }^{\mathrm{a}}$, Jens Möller ${ }^{\mathrm{b}}$, Viola Vogel ${ }^{\mathrm{b}}$, and Rupert Konradi§^a \\ §SCS Poster Prize Winner
}

\begin{abstract}
Surface fouling, i.e. the non-specific surface adhesion of proteins, bacteria and higher organisms, poses a severe problem in many areas ranging from modern diagnostic and therapeutic medical devices to food processing and food wrapping technology to corrosion prevention and marine technology. One approach to address these problems is to coat surfaces with nonfouling polymers. The properties of a new class of nonfouling polymer coatings made from poly(2-methyl-2-oxazoline) (PMOXA) were investigated here in comparison with the most frequently used polymer in this context, poly(ethylene glycol) (PEG). Both polymers were side-chain grafted onto a polycationic poly-L-lysine (PLL) backbone. The PMOXA graft copolymers spontaneously self-assembled to form monolayers on negatively charged surfaces. PMOXA surface coatings were as efficient as PEG-based coatings in suppressing protein and bacterial adsorption. The minimal number of side chain monomer units per surface area that are needed to obtain fully resistant surfaces was lower though for PMOXA than for PEG graft copolymers as a result of the higher molecular weight of the PMOXA monomer unit.
\end{abstract}

Keywords: Biointerface science $\cdot$ Nonfouling $\cdot$ Polymer brush $\cdot$ Poly(ethylene glycol) $\cdot$ Poly(2-methyl-2-oxazoline)

\section{Introduction}

So-called nonfouling, bioinert or 'stealth' polymers are increasingly being used for the fabrication of bulk hydrogel materials for application e.g. in tissue engineering, ${ }^{[1]}$ for the decoration of liposomes, particles and capsules, ${ }^{[2.3]}$ and for the modification of biomedical implant ${ }^{[4]}$ and biosensor surfaces. ${ }^{[5]}$ Typically, these polymers are strongly hydrated and once grafted to an interface at

${ }^{\star}$ Correspondence: Dr. R. Konradia

Tel.: + 41446336168

Fax: + 41446331027

E-mail: rupert.konradi@mat.ethz.ch

aLaboratory for Surface Science and Technology

bLaboratory for Biologically Oriented Materials

Department of Materials, ETH Zürich

ETH-Hönggerberg

Wolfgang-Pauli-Strasse 10

$\mathrm{CH}-8093$ Zürich sufficient density, they oppose non-specific adsorption of proteins and other biological molecules by entropic and osmotic repulsion. ${ }^{[6-8]}$ Since protein adsorption is usually the first step in a cascade of biological events, nonfouling polymers, depending on the exact biological environment, can serve for instance to lower the background noise of biosensors, ${ }^{[5]}$ to slow down the phagocytosis of drug delivery vehicles, ${ }^{[2]}$ and to prevent blood clot formation, triggering of the foreign body response, and implantassociated infections. ${ }^{[9]}$

Empirical design criteria for nonfouling polymers have been proposed.[10,11] Accordingly, they should be hydrophilic, electrically neutral and should have hydrogenbond acceptor but no donor functionality. While today, poly(ethylene glycol) (PEG) is routinely used as nonfouling polymer, ${ }^{[12]}$ material scientists, motivated by certain limitations in PEG stability (autoxidation) and functionality, ${ }^{[13,14]}$ have set out to identify alternative polymers including for instance polysaccharides, ${ }^{[15]}$ zwitterionic ${ }^{[16-18]}$ and peptidomimetic polymers ${ }^{[19]}$ as a substitute for PEG. In the past decade, poly(2-methyl-2-oxazoline) (PMOXA), a very hydrophilic and nonionic polymer, has attracted increasing interest for use in biological applications and showed very promising properties. For instance, PMOXA-enzyme conjugates retained high enzyme activity, ${ }^{[20]}$ PMOXA-decorated liposomes showed long circulation times in blood, ${ }^{[21,22]}$ transmembrane receptors could be reconstituted into supported lipid bilayer membranes assembled from PMOXAlipopolymers ${ }^{[23]}$ and vesicles consisting of amphiphilic PMOXA-terminal triblock copolymers allowed for the incorporation of functional channel-forming membrane proteins $^{[24]}$ and were non-cytotoxic against human monocytic THP-1 cells. ${ }^{[25]}$

The structure of PMOXA is composed of a poly(ethylene imine) backbone and amide-bonded acetyl groups (see Fig. 1 on the right). Thus, on one hand, PMOXA resembles PEG in having a heteroatom separated by an ethylene unit in the backbone. On the other hand, PMOXA is isomeric to poly(homoalanine) and has a peptidelike structure. PMOXA of well-controlled molecular weight and polydispersity can readily be synthesized by living cationic polymerization of 2-methyl-2-oxazoline. Moreover, functional initiators, terminating agents and in particular functional comonomers can readily be incorporated in a controlled sequence to produce PMOXA with well-defined functionality.

In this paper we investigated for the first time the ability of PMOXA-modified solid substrates to resist the adhesion of proteins and bacteria in quantitative comparison to the nonfouling properties of PEG. To this 


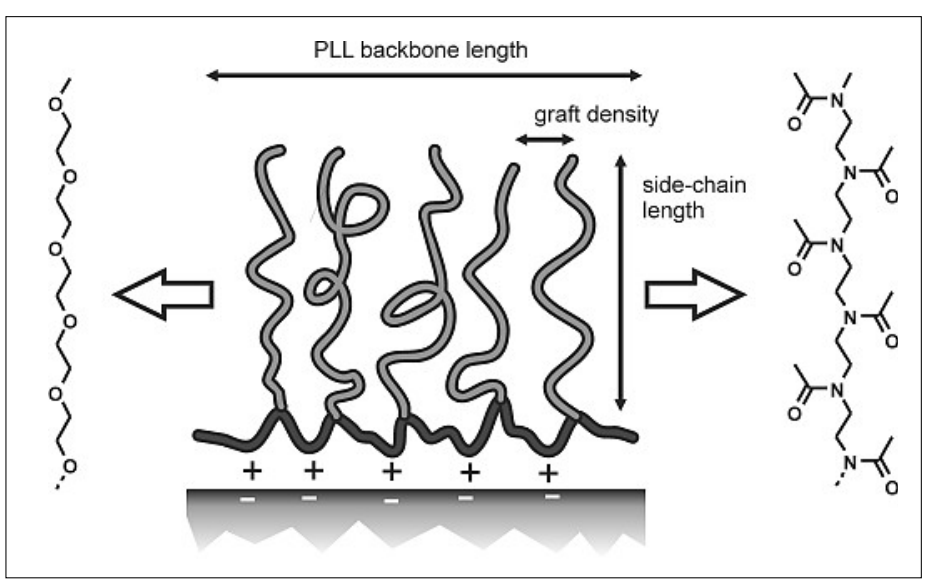

Fig. 1. General schematic illustration of the interfacial architecture of PLL$g$-PMOXA or PLL-g-PEG comb copolymers self-assembled on negatively charged surfaces. Variables in the molecular design are indicated. Note that the stretching of the side-chains is exaggerated. end, we designed PMOXA graft copolymers by close analogy to the thoroughly studied poly(L-lysine)-graft-poly(ethylene glycol) (PLL-g-PEG) system (see Fig. 1), both comprising a polycationic PLL backbone that serves as a multiple electrostatic anchor. ${ }^{[26-30]}$ The copolymers spontaneously self-assemble onto negatively charged substrates such as glass, various metal oxides and tissue culture polystyrene. The side chains, depending on the length and grafting density, form a polymer brush since, during adsorption, one half-space becomes inaccessible. PLL- $g$-PEG coatings of optimized architecture have proved very effective in reducing protein and bacteria fouling. [28,31]

\section{Materials and Methods}

PLL- $g$-PMOXA was synthesized in three steps as previously described (Scheme).[32] Briefly, the cationic ring opening polymerization of 2-methyl-2-oxazoline was initiated with methyl triflate at an initiator to monomer ratio of 1:50. The living polymerization was terminated with $\mathrm{Na}_{2} \mathrm{CO}_{3}$ /water to yield hydroxyl-terminated PMOXA. After purification by dialysis (molecular weight cut off, MWCO = $1000 \mathrm{Da}$ ) and lyophilization, the polymer was azeotropically dried in acetonitrile and directly reacted with an excess of glutaric anhydride terminal hydroxyl group into a carboxylic acid function. ${ }^{[21,22]}$ The carboxy-functional PMOXA was again purified by dialysis and lyophilization and characterized by ${ }^{1} \mathrm{H}-$ NMR. By comparison of the integrals from the methylene group adjacent to the esterbonded oxygen and the methylene groups in the backbone, the degree of polymerization DP was found to be approximately 56 , close to the expected value of 50 and corresponding to a number average molecular weight in the presence of pyridine to convert the of approximately $5000 \mathrm{~g} / \mathrm{mol}$. Finally, using water-soluble carbodiimide chemistry (EDC/Sulfo-NHS), carboxy-terminated PMOXA was grafted onto poly(L-lysine) hydrobromide (PLL) in Hepes I buffer (10 mmol/l Hepes adjusted with $\mathrm{NaOH}$ to $\mathrm{pH}$ $=7.4$ ). By variation of the molar ratio of PMOXA-COOH and PLL a set of PLL- $g$ PMOXA with different densities of grafted PMOXA side chains was prepared. To remove any low molecular weight coupling agents and unreacted PMOXA, the graft copolymers were purified by dialysis through 12000-14000 Da MWCO tubing, followed by lyophilization and ${ }^{1} \mathrm{H}-\mathrm{NMR}$ characterization. The signal originating from the methylene unit adjacent to the lysine amine group slightly shifts upon conversion of the amine into an amide and was used to determine the grafting density $\alpha$ defined as the number of PMOXA-grafted lysine units divided by the total number of lysine units. grafting density of PLL- $g$-PEG is defined analogously.

Optical waveguide lightmode spectroscopy (OWLS 110 and OWLS 120, Microvacuum Ltd., Budapest, Hungary) was used tion in situ and in real time as previously described. ${ }^{228,32,33]}$

E. coli FimH-j96 K12 bacteria were used to study microbial adhesion. The recombinant strain was constructed as previously described, ${ }^{[34-36]}$ and is morphologically identical to the E. coli $\mathrm{K} 12$ wild-type strain but expresses fully functional type 1 fimbriae at all times. The bacteria were grown overnight at $37{ }^{\circ} \mathrm{C}$ in SB growth medium containing $100 \mu \mathrm{g} / \mathrm{ml}$ ampicillin and $25 \mu \mathrm{g} /$ $\mathrm{ml}$ chloramphenicol to specifically select for the recombinant bacteria. The bacteria were collected by centrifugation, washed twice in Hepes II buffer $(10 \mathrm{mmol} / \mathrm{l}$ Hepes, $150 \mathrm{mmol} / \mathrm{l} \mathrm{NaCl}$, adjusted with $\mathrm{NaOH}$ to $\mathrm{pH}=7.4$ ) and adjusted to a concentration of $10^{9} \mathrm{cfu} / \mathrm{ml}$ in Hepes II. Microscopy slides were sputter-coated with a $15-\mathrm{nm}$ thick $\mathrm{Nb}_{2} \mathrm{O}_{5}$ layer (Leybold direct current magnetron Z600 sputtering unit; PSI, Villigen, Switzerland) and cleaned in the same way as the OWLS waveguides. For surface modification, they were placed in a $0.1 \mathrm{mg} /$ $\mathrm{ml}$ solution of the respective polymer in Hepes II for $\geq 2 \mathrm{~h}$ and subsequently rinsed with deionized water and dried in a stream of nitrogen. The sample surfaces were allowed to rehydrate in Hepes II for $30 \mathrm{~min}$ at room temperature before incubation with bacteria for $20 \mathrm{~min}$ at $37^{\circ} \mathrm{C}$. After incubation, the surfaces were extensively washed $\alpha$ was varied between 0.05 and 0.77 . The to study polymer and serum surface adsorp-

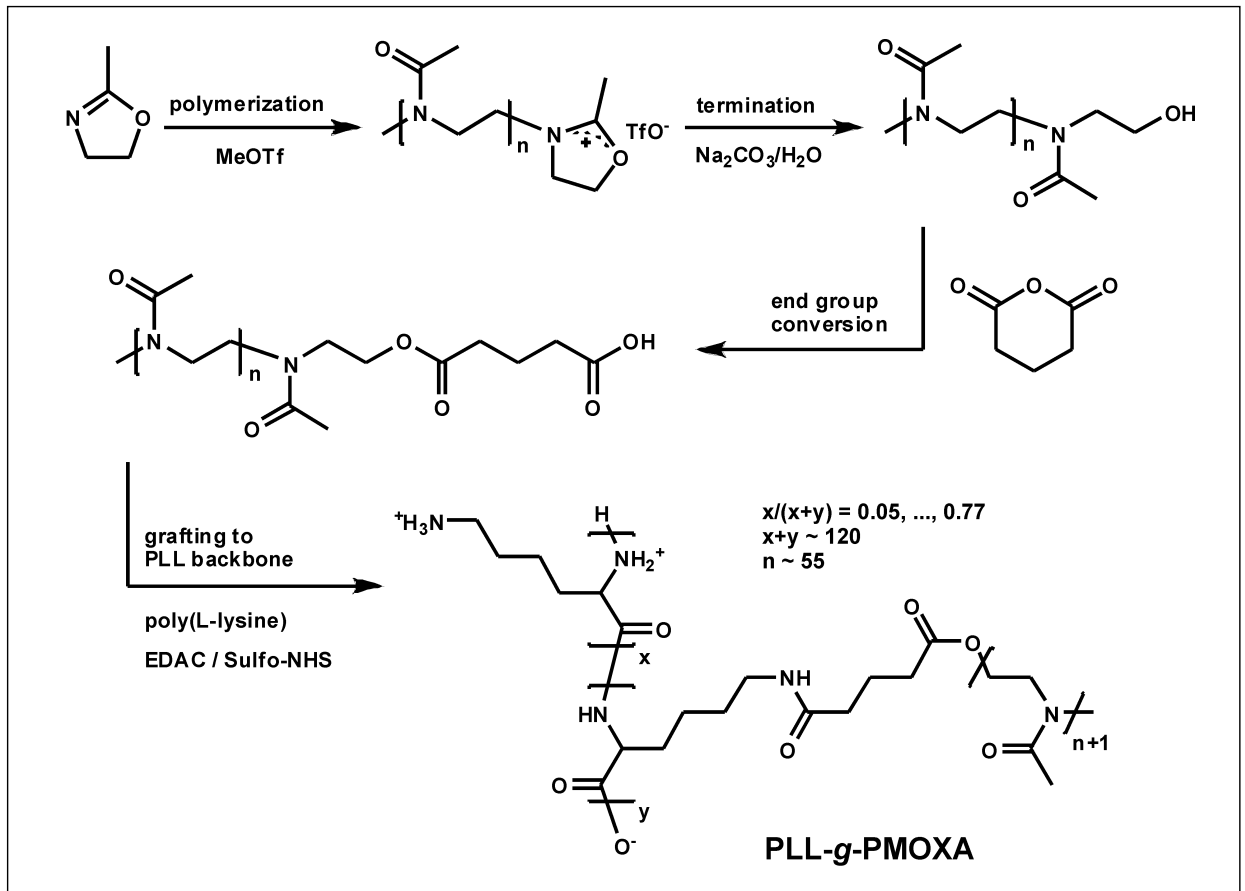

Scheme. Synthetic strategy for the preparation of PLL-g-PMOXA 
with Hepes II to remove planktonic cells. Adhering bacteria were analyzed by phase contrast light microscopy (Nikon TE2000E microscope, Nikon AG). Each sample was prepared in triplicate and ten images at $40 \times$ magnification were taken from each sample for statistical analysis. Data acquisition and analysis was carried out using SimplePCI (Hamamatsu Corporation) and MetaMorph (Universal Imaging Corporation) software.

\section{Results and Discussion}

First, we studied the surface self-assembly of PLL-g-PMOXA graft copolymers and the resistance of the modified surfaces to the adsorption of serum proteins in situ and in real time by OWLS. Typical measurements for PLL- $g$-PMOXA of different grafting densities are shown in Fig. 2. To this end, niobium oxide coated waveguides were equilibrated with Hepes II buffer until a stable baseline was obtained. Then, a $0.1 \mathrm{mg} / \mathrm{ml}$ solution of PLL- $g$-PMOXA in Hepes II was injected in stop flow mode. For grafting densities up to $\alpha=0.59$, a steep rise in the adsorbed mass followed by a further slow increase for up to about $2 \mathrm{~h}$ was observed (Fig. 2, left panel). The polymer solution was replenished to ensure that polymer depletion close to the surface did not limit the adsorption process. Subsequent rinsing with buffer did not result in any mass loss, indicating that the PLL$g$-PMOXA monolayer was stably and, on an experimentally accessible timescale, irreversibly adsorbed. At this point the adsorbed polymer mass was determined as exemplarily indicated in Fig. 2 for $\alpha=0.59$. Then, the PMOXA-modified surface was incubated with full human serum (Control serum, Precinorm ${ }^{\circledR}$ U, LOT: 171 074-01, reconstituted in ultrapure water) for $15 \mathrm{~min}$ and subsequently rinsed with buffer again (Fig. 2, right panel). The steep rise in the curve upon exposure to serum originates from the dramatic increase in the solution refractive index due to the high protein concentration and does not reflect a true increase in mass. The serum adsorbed mass was determined as indicated in Fig. 2, right panel, by comparison of the adsorbed mass in buffer solution before and after the polymer monolayer had been exposed to serum. The highest polymer adsorbed mass of $>200$ $\mathrm{ng} / \mathrm{cm}^{2}$ was found for PLL- $g$-PMOXA of medium grafting density (see the curve for $\alpha=0.22$ ). The corresponding serum adsorbed mass was below the detection limit of the instrument $\left(\sim 1-2 \mathrm{ng} / \mathrm{cm}^{2}\right)$. ${ }^{[33]}$ Both at low and high grafting densities (see the curves for $\alpha=0.10$ and $\alpha=0.59$ ), less graft copolymer was deposited and significant amounts of serum proteins adsorbed. Strikingly, at very high grafting densities (see the curve for $\alpha=0.77$ ), when almost

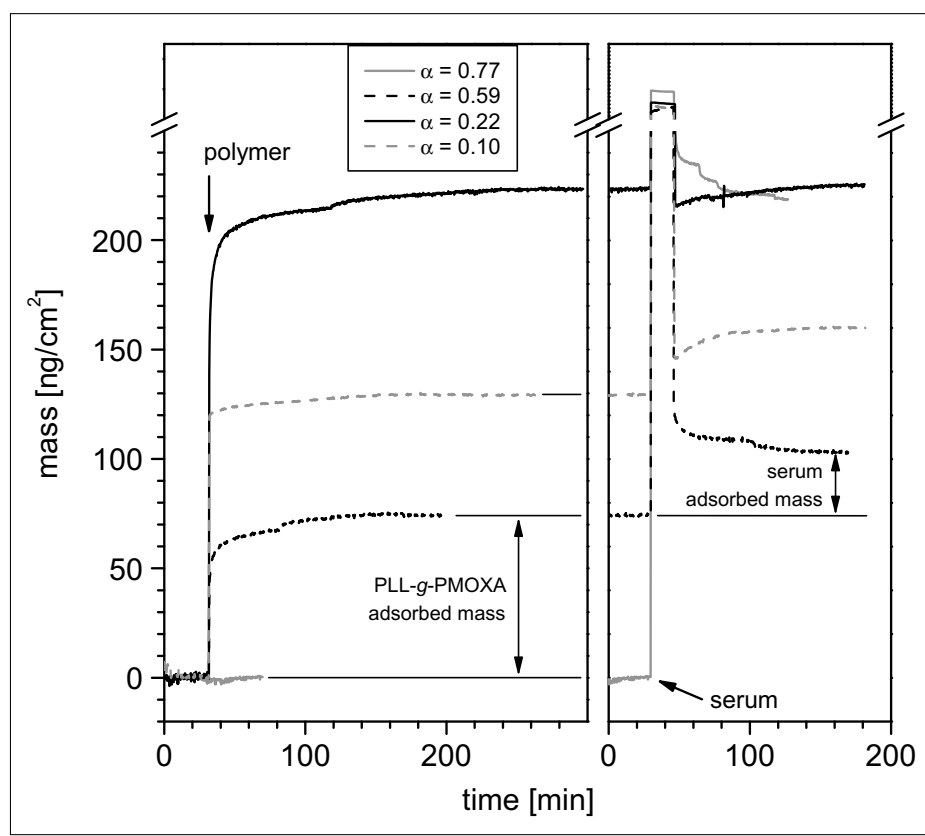

Fig. 2. Exemplary in situ optical waveguide lightmode spectroscopy (OWLS) measurements for PLL-g-PMOXA of grafting densities $\alpha=0.10$, $0.22,0.59$ and 0.77 : First, negatively charged niobium oxide coated waveguides are incubated with PLL-g-PMOXA in physiological buffer. The increase in adsorbed mass due to the graft copolymer self-assembly is recorded as a function of time (left panel). After rinsing with buffer, the polymer-modified surfaces are incubated with full human serum for $15 \mathrm{~min}$ (right panel). The steep rise in mass after serum injection is mostly due to a dramatic increase in refractive index and cannot be assigned to a mass value. Only after rinsing with buffer again, the serum adsorbed mass can be determined by comparing the mass before and after serum injection. The reading of the polymer and serum adsorbed mass is exemplarily shown for PLL-g-PMOXA of grafting density $\alpha=0.59$.

every lysine unit carries a PMOXA side chain, polymer binding to the negatively charged surface was completely suppressed while, subsequently, a monolayer of serum proteins with a mass $>200 \mathrm{ng} / \mathrm{cm}^{2}$ adsorbed onto the surface, similar to the case of a blank niobium oxide surface.

Similarly, a set of nine PLL- $g$-PMOXA of different grafting densities and pure PLL were investigated and each experiment was repeated three times. In Fig. 3 we compare the polymer and serum adsorbed masses with previously published data for analogous experiments where PLL- $g$-PEG of different grafting densities and with three different PEG side chain lengths, PEG(1kDa), PEG(2kDa) and PEG(5kDa), were employed. ${ }^{[28]}$ Since both the interfacial molecular architecture and the analytical technique were the same, this study allows for a direct and quantitative comparison of the nonfouling properties of PEG and PMOXA. In Fig. $3 \mathrm{a}$ and $\mathrm{b}$ the polymer and serum adsorbed masses for PLL- $g$-PMOXA(5kDa), PLL- $g$ PEG(1kDa), PLL- $g$-PEG(2kDa) and PLL$g$-PEG $(5 \mathrm{kDa})$ are plotted as a function of the grafting density, respectively. The adsorbed copolymer mass linearly increases with grafting density for PLL- $g$-PEG $(1 \mathrm{kDa})$ (Fig. 3a). A similar behavior is found for all other polymers at low to medium grafting densities up to $\alpha \sim 0.25-0.3$. At the highest grafting density of PLL- $g$-PEG( $2 \mathrm{kDa})$ and PLL- $g$-PEG(5kDa), however, a decrease in adsorbed polymer mass is observed. This effect is even more pronounced for PLL$g$-PMOXA $(5 \mathrm{kDa})$ where the adsorbed polymer mass as a function of the grafting density clearly passes through a maximum and at extremely high grafting density ( $\alpha$ $=0.77$ ) becomes zero. The adsorbed serum mass (Fig. 3b) shows an inverse behavior: In the low grafting density regime, serum adsorption decreases with increasing grafting density and increasing polymer mass. At the highest polymer coverages, at medium grafting densities, serum adsorption is essentially zero. At high grafting densities, where PLL- $g$-PEG(5kDa) and PLL- $g$ PMOXA $(5 \mathrm{kDa})$ yield significantly lower adsorbed polymer masses, serum adsorption is restored. Fortunately, the observed minimum in serum adsorbed mass is rather broad, thus, the exact grafting density is not crucial to obtain highly protein-repelling surfaces.

To get a better understanding of the interfacial architecture leading to these trends, we have calculated the number of lysine monomer units per surface area, $n_{L y s}$, and the number of PEG or PMOXA side chains per surface area, $n_{P E G}$ or $n_{P M O X A}$, by 

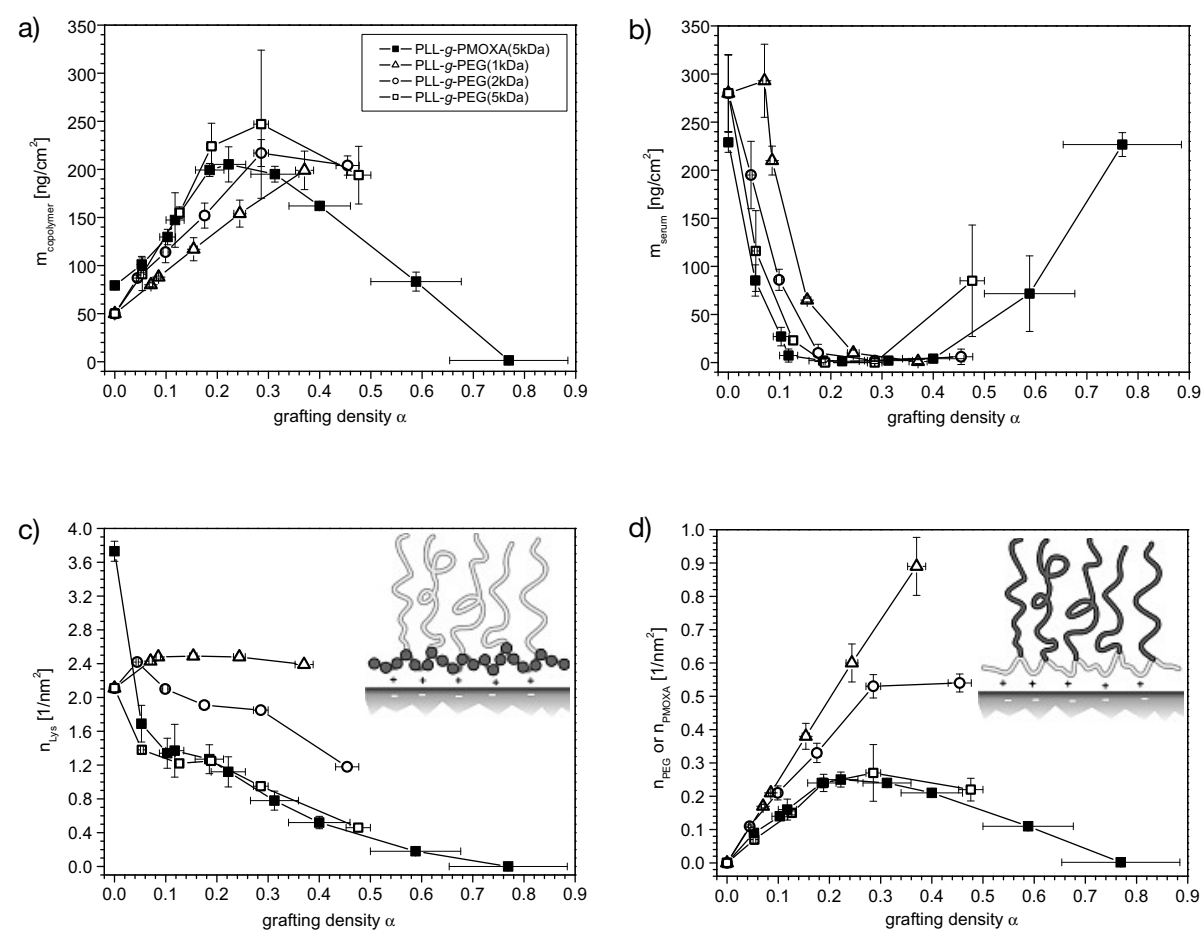

Fig. 3. Comparison of the adsorbed polymer mass (a), the adsorbed serum mass (b), the surface density of lysine monomer units (c) and the surface density of PEG or PMOXA side chains (d) as a function of the grafting density $\alpha$ for PLL-g-PMOXA(5kDa) (ם), PLL-g-PEG(1kDa) ( $\triangle$ ), PLL-gPEG(2kDa) (O), and PLL-g-PEG(5kDa) ( $\square)$. $\alpha=0$ corresponds to pure PLL. Values for PLL-g-PEG are taken from Pasche et al. ${ }^{[28]}$

using the adsorbed polymer mass $m_{\text {pol }}$, the grafting ratio $g$, and the side chain number average molecular weight $M_{P E G}$ or $M_{P M O X A}$ according to

$$
n_{\text {Lys }}=\frac{m_{p o l}}{\left(M_{L y s}+\frac{M_{P M O X A}}{g}\right)} \cdot N_{A}
$$

$$
n_{P M O X A}=\frac{m_{p o l}}{\left(M_{L y s} \cdot g\right)+M_{P M O X A}} \cdot N_{A}
$$

with the molecular weight of the lysine monomer unit $M_{L y s}$ and Avogadro's number $N_{A}$. Fig. $3 \mathrm{c}$ and d show $n_{L y S}$ and $n_{P M O X A}$ with $n_{P E G}$, respectively, as a function of the grafting density. With the exception of pure PLL, ${ }^{[37]} n_{\text {Lvs }}$ more or less stays constant up to $\alpha \sim 0.2$ for all graft copolymers. In this regime, $n_{P M O X A}, n_{P E G}$ and $m_{\text {copolymer }}$ for all copolymers increase approximately linearly with grafting density since at constant backbone coverage essentially every added side chain equally contributes to the adsorbed mass. For PLL- $g$-PEG $(1 \mathrm{kDa})$ this regime extents up to the highest grafting density studied since the rather short 1 kDa PEG side chains do not impose sufficient crowding to significantly reduce the lysine surface density. In contrast, for PLL-
$g$-PEG(2kDa) and in particular for PLL- $g$ PEG(5kDa) and PLL- $g$-PMOXA(5kDa), $n_{\text {Lys }}$ monotonously decreases at higher grafting densities. At least for the latter two copolymers, this eventually leads to a decrease in $n_{P M O X A}$ and $n_{P E G}$ and, concomitantly, to a decrease in $m_{\text {copolymer }}$. Several factors limit the graft copolymer adsorption at high grafting densities: First, upon adsorption, one half space becomes inaccessible for the copolymer molecules, leading, at sufficiently high grafting density, to side chain stretching and a loss in conformational entropy. Second, the adsorption enthalpy associated with the multiple electrostatic attractions between polymer backbone and surface declines with increasing grafting density since positive backbone charges are consumed by converting the amine into an amide during the grafting reaction. Third, the copolymer surface adsorption and side chain brush formation is a self-limiting 'grafting to' process that becomes more and more kinetically hindered due to an increasing diffusion barrier imposed by already adsorbed copolymer molecules. ${ }^{[38,39]}$ This effect will be more pronounced at higher densities of grafted side chains. Finally, with increasing grafting density and length of the side chains, the copolymer backbone becomes less and less flexible, impeding surface rearrangement of the backbone segments. ${ }^{[30,40]}$ This may lead to inhomogeneously and incompletely covered surfaces.
At the highest grafting density of PLL- $g$ PMOXA $(5 \mathrm{kDa})$, at $\alpha=0.77$, the graft copolymer molecules form a molecular brush in solution and adsorption is fully inhibited.

The extent of serum adsorption not only depends on the density of grafted copolymer side chains, but also on their length and type of chemistry (see Fig. 3b). For PLL- $g$ PEG, it has been found that the longer the PEG chains, the lower the grafting density needed to achieve resistance to serum adsorption. ${ }^{[28]}$ Therefore, it is of interest to plot the serum adsorbed mass as a function of the monomer surface density, that is, the number of ethylene glycol or methyloxazoline monomer units per surface area, $n_{E G}$ or $n_{M O X A}$. This quantity increases with both, density and length of grafted chains, and can be readily calculated from $n_{P E G}$ or $n_{P M O X A}$ and the molecular weight of the monomer unit $M_{E G}$ or $M_{M O X A}$ according to: ${ }^{[28]}$

$$
n_{M O X A}=n_{P M O X A} \cdot \frac{M_{P M O X A}}{M_{M O X A}}
$$

The corresponding graph is shown in Fig. 4. Irrespective of the PEG chain length, all PEG-based copolymers fall on a master curve, illustrating that indeed the combination of PEG chain grafting density and length, as reflected in the monomer surface density, controls in a predictive manner the nonfouling behavior. In contrast, the curve for the PMOXA-based copolymers has a very similar shape but it is shifted to lower monomer surface densities. Whereas full protein resistance is obtained at a critical monomer surface density of approximately $n_{E G} \geq 20$ units $/ \mathrm{nm}^{2}$ in the case of PLL- $g$ PEG, a critical density of $n_{M O X A} \approx 10-12$ units $/ \mathrm{nm}^{2}$ was found to be sufficient in the case of PLL- $g$-PMOXA. We attribute this difference to the fact that the molecular weights of the two monomer units differ by almost a factor of two (85 vs. $44 \mathrm{~g} / \mathrm{mol}$ ). Hence, the contribution of a MOXA monomer unit to the entropic and osmotic penalty opposing polymer brush compression upon protein adsorption will be higher than the contribution of an EG monomer unit.

To test the biological relevance of our findings, we finally compared the resistance of PEG and PMOXA-modified surfaces to bacterial adhesion. To this end, microscopy slides with a thin niobium oxide coating were treated with PLL- $g$-PEG(2kDa) and PLL- $g$-PMOXA $(5 \mathrm{kDa})$ of optimal grafting densities $(\alpha=0.29$ and 0.22 , respectively), and subsequently incubated for 20 min with $10^{9} \mathrm{cfu} / \mathrm{ml}$ E. coli $\mathrm{K} 12$ bacteria in physiological buffer at $37^{\circ} \mathrm{C}$. After the bacterial suspension was thoroughly exchanged for pure buffer, optical phase contrast micrographs were taken from the hydrated sam- 


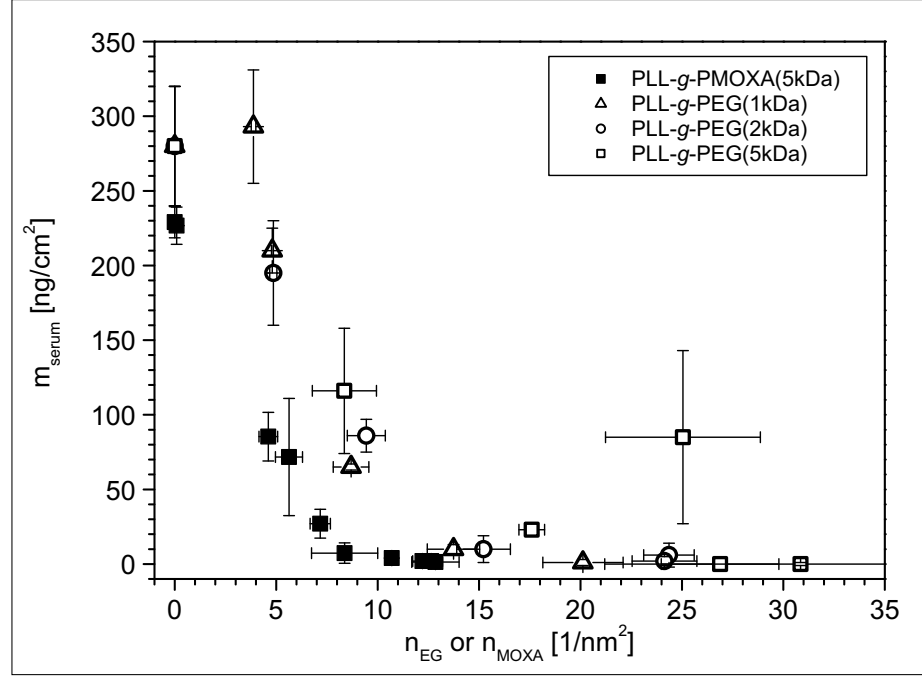

Fig. 4. Serum adsorbed mass as a function of the side chain monomer surface density $n_{E G}$ (number of ethylene glycol monomer units per surface area) or $n_{M O X A}$ (number of 2-methyl-2-oxazoline monomer units per surface area) for PLL-g-PMOXA(5kDa) (ם), PLL-g-PEG(1kDa) ( $\triangle$ ), PLL$g$-PEG(2kDa) (O), and PLL-g-PEG(5kDa) ( $\square$ ). Values for PLL-g-PEG are taken from Pasche et al.[28] ples and the number of adherent bacteria was counted. In Fig. 5a, typical micrographs for the blank niobium oxide substrate, the bare PLL backbone coated substrate, and for the two graft copolymer-modified surfaces are depicted. Fig. 5b shows the quantitative evaluation of the micrographs. A large number of bacteria adsorbed onto the blank substrate even though both, E. coli bacteria and the niobium oxide substrate, carry a net negative charge. Most likely this attraction predominantly resulted from hydrophobic interactions. ${ }^{[41]}$ When the substrate was modified with the bare PLL backbone, bacterial adhesion increased by more than a factor of two due to additional electrostatic attraction. In contrast, coating of the surface with either PLL- $g$-PEG or PLL- $g$ PMOXA leads to a tremendous reduction in bacterial adhesion by $>99 \%$ compared to the blank substrate. This demonstrates that bacterial surface colonization can be prevented by both PEG and PMOXA-based surface coatings, to a similar extent. The few bacteria that were still visible in these cases did not firmly stick to the surface but were (partially) still mobile and originated from the edges of the wells where bacteria, even by excessive washing, could not be fully removed. We regard this reduction of bacterial adhesion by 2-3 orders of magnitude as the resolution limit of the assay. Higher log-reductions, as they are typically obtained by plating of planktonic bacteria, were not accessible.

\section{Conclusions}

We have fabricated graft copolymers comprising a polycationic backbone and two chemically distinctively different nonfouling side chains: poly(ethylene glycol) (PEG) and poly(2-methyl-2-oxazoline) (PMOXA). By choosing the analogous molecular architecture and by employing the same analytical methods we quantitatively compared the nonfouling properties of the two side chain polymers with regard to proteins and bacteria. Using optical waveguide lightmode spectroscopy we found that both types of graft copolymers similarly selfassemble onto negatively charged metal oxide surfaces to form stable monolayers with the maximal PMOXA or PEG surface density being obtained for copolymers of medium density of grafted side chains. At higher densities, steric, energetic and kinetic effects along with the reduced copolymer flexibility increasingly opposed polymer adsorption. At very high grafting densities, when about three out of four backbone segments carry a PMOXA side chain, polymer adsorption was fully inhibited. At optimal grafting densities, serum adsorption, within the resolution limit of OWLS ( 1-2 $\mathrm{ng}$ / $\mathrm{cm}^{2}$ ), could be fully suppressed by both types of copolymers. The minimal number of side chain monomer units per surface area needed to obtain fully resistant surfaces was lower for PMOXA than for PEG graft copolymers as a result of the higher molecular weight of the PMOXA monomer unit. PMOXA and PEG based copolymers also showed an equally high capability to prevent bacterial surface adhesion. In both cases, $E$. coli adhesion was reduced to $<1 \%$ compared to the blank substrate, thus approaching the detection limit of the optical microscopy assay. In view of the well-controlled and versatile polymerization chemis-

\section{a)}

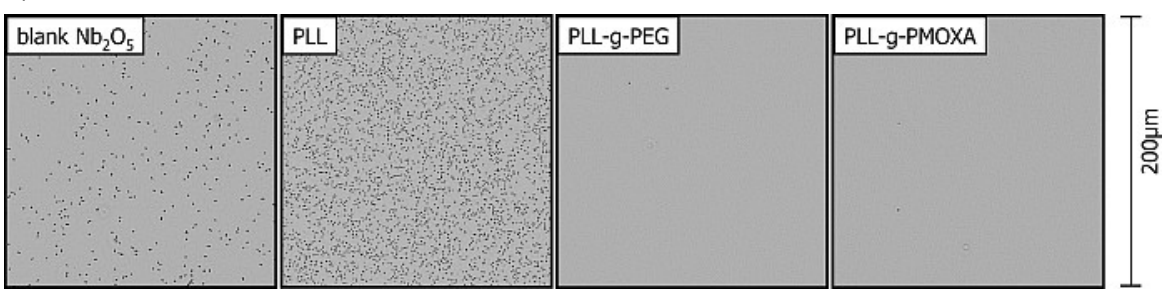

b)

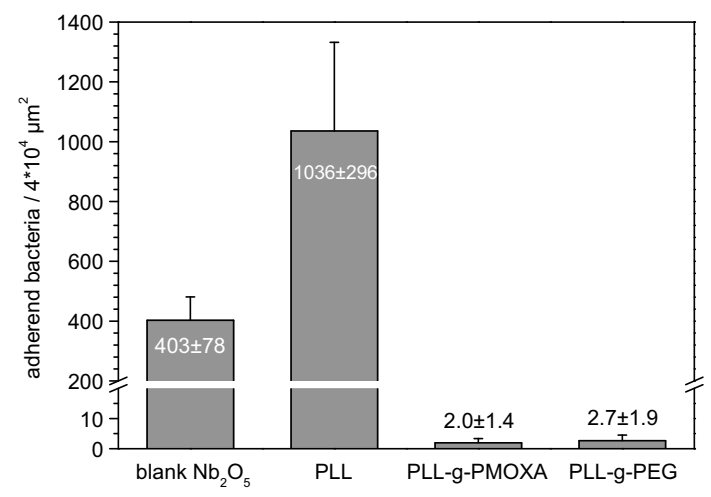

Fig. 5. a) Exemplary optical phase contrast micrographs of blank, PLL, PLL-g-PEG $(\alpha=0.29)$ and PLL-g-PMOXA ( $\alpha=0.22)$ modified $\mathrm{Nb}_{2} \mathrm{O}_{5}$-coated microscopy slides after a 20 min exposure to $10^{9}$ $\mathrm{cfu} / \mathrm{ml}$ E. coli K12 at $37{ }^{\circ} \mathrm{C}$ in physiological buffer. b) Quantitative evaluation of the micrographs reveals a $>99 \%$ reduction in bacterial adhesion for both PLL- $g$-PEG and PLL-g-PMOXA modified surfaces in comparison to the blank niobium oxide substrate. 
try of 2-oxazolines, that allows for the facile synthesis of functional polyoxazolines, and the herein reported excellent nonfouling properties of PMOXA, we believe that the PMOXA technology holds great promise for the fabrication of sophisticated nonfouling surface coatings with defined molecular architecture and functionality for the use in biointerphase science and the development of novel therapeutic and diagnostic medical devices.

\section{Acknowledgments}

We thank Andreas Mühlebach for valuable discussions. R.K. is grateful to the Alexandervon-Humboldt foundation for a Feodor-Lynen grant. This research was financially supported by Ciba Specialty Chemicals.

\section{Received: February 25, 2008}

[1] M. P. Lutolf, J. A. Hubbell, Nat. Biotechnol. 2005, 23, 47.

[2] M. C. Woodle, Adv. Drug Delivery Rev. 1998, 32, 139 .

[3] T. G. Park, J. H. Jeong, S. W. Kim, $A d v$. Drug. Deliv. Rev. 2006, 58, 467.

[4] B. D. Ratner, S. J. Bryant, Annu. Rev. Biomed. Eng. 2004, 6, 41.

[5] D. Falconnet, G. Csucs, H. M. Grandin, M. Textor, Biomaterials 2006, 27, 3044.

[6] A. Halperin, G. Fragneto, A. Schollier, M. Sferrazza, Langmuir 2007, 23, 10603.

[7] F. Fang, I. Szleifer, Langmuir 2002, 18, 5497.

[8] S. I. Jeon, J. H. Lee, J. D. Andrade, P. G. De Gennes, J. Colloid Interface Sci. 1991, $142,149$.

[9] G. Harris Llinos, R. G. Richards, Injury 2006, 37 Suppl 2, S3.

[10] E. W. Merrill, Ann. N Y Acad. Sci. 1987, 516, 196.

[11] R. G. Chapman, E. Ostuni, M. N. Liang, G. Meluleni, E. Kim, L. Yan, G. Pier, H. S. Warren, G. M. Whitesides, Langmuir 2001, 17, 1225 .

[12] J. M. Harris, S. Zalipsky, 'Polyethylene Glycol: Chemistry and Biological Applications', Am. Chem. Soc., Washington, DC, 1997, Vol. 680.

[13] M. Donbrow, in 'Nonionic Surfactants: Physical Chemistry', Ed. M. J. Schick, Marcel Dekker, New York, 1987, Vol. 23.

[14] M. Shen, L. Martinson, M. S. Wagner, D. G. Castner, B. D. Ratner, T. A. Horbett, $J$. Biomater. Sci., Polym. Ed. 2002, 13, 367.

[15] N. B. Holland, Y. Qiu, M. Ruegsegger, R. E. Marchant, Nature 1998, 392, 799.

[16] S. L. West, J. P. Salvage, E. J. Lobb, S. P. Armes, N. C. Billingham, A. L. Lewis, G. W. Hanlon, A. W. Lloyd, Biomaterials 2003, 25, 1195 .

[17] Z. Zhang, S. Chen, Y. Chang, S. Jiang, $J$. Phys. Chem. B 2006, 110, 10799.

[18] W. Feng, J. L. Brash, S. Zhu, Biomaterials 2006, $27,847$.

[19] A. R. Statz, R. J. Meagher, A. E. Barron, P. B. Messersmith, J. Am. Chem. Soc. 2005, 127, 7972

[20] M. Miyamoto, K. Naka, M. Shiozaki, Y. Chujo, T. Saegusa, Macromolecules 1990, 23, 3201.
[21] M. C. Woodle, C. M. Engbers, S. Zalipsky, Bioconj. Chem. 1994, 5, 493.

[22] S. Zalipsky, C. B. Hansen, J. M. Oaks, T. M. Allen, J. Pharm. Sci. 1996, 85, 133.

[23] O. Purrucker, A. Förtig, R. Jordan, M Tanaka, ChemPhysChem 2004, 5, 327.

[24] C. Nardin, J. Widmer, M. Winterhalter, W. Meier, Europ. Phys. J. E 2001, 4, 403.

[25] P. Broz, S. M. Benito, C. Saw, P. Burger, H. Heider, M. Pfisterer, S. Marsch, W. Meier, P. J. Hunziker, Controlled Release 2005, 102, 475 .

[26] G. L. Kenausis, J. Vörös, D. L. Elbert, N. Huang, R. Hofer, L. Ruiz-Taylor, M Textor, J. A. Hubbell, N. D. Spencer, J. Phys. Chem. B 2000, 104, 3298.

[27] N.-P. Huang, R. Michel, J. Vörös, M. Textor, R. Hofer, A. Rossi, D. L. Elbert, J. A. Hubbell, N. D. Spencer, Langmuir 2001, 17, 489.

[28] S. Pasche, S. M. De Paul, J. Vörös, N. D. Spencer, M. Textor, Langmuir 2003, 19, 9216

[29] S. Pasche, M. Textor, L. Meagher, N. D. Spencer, H. J. Griesser, Langmuir 2005, 21, 6508.

[30] L. Feuz, P. Strunz, T. Geue, M. Textor, O. Borisov, Europ. Phys. J. E 2007, 23, 237.

[31] L. G. Harris, S. Tosatti, M. Wieland, M. Textor, R. G. Richards, Biomaterials 2004, 25, 4135.

[32] R. Konradi, B. Pidhatika, A. Muehlebach, M. Textor, Langmuir, 2008, 24, 613 .

[33] J. Vörös, J. J. Ramsden, G. Csucs, I Szendro, S. M. De Paul, M. Textor, N. D Spencer, Biomaterials 2002, 23, 3699.

[34] W. E. Thomas, E. Trintchina, M. Forero, V. Vogel, E. V. Sokurenko, Cell 2002, 109, 913.

[35] I. C. Blomfield, V. Vaughn, R. F. Rest, B. I. Eisenstein, Mol. Microbiol. 1991, 5 1447.

[36] I. C. Blomfield, M. S. McClain, B. I Eisenstein, Mol. Microbiol. 1991, 5, 1439.

[37] A somewhat higher lysine surface density was observed for pure PLL than for some of the copolymers at low grafting density. We assume that this is due to loop formation during PLL adsorption while in case of the graft copolymers, loop formation is opposed by side chain crowding. In an angle-resolved XPS study of the interfacial architecture of PLL- $g$ PEG it was found that the PLL backbone within the accuracy of the technique lies flat on the substrate with the PEG side chains protruding into solution. ${ }^{[27]}$ Moreover, the adsorbed amount of polyelectrolytes strongly depends on the ionic strength in solution. At higher ionic strengths, an increasing number of charged segments become screened and loop formation is favored. In the previous study on PLL- $g$-PEG, the polymers and PLL were adsorbed from $10 \mathrm{mmol} / \mathrm{L}$ Hepes solution, ${ }^{[28]}$ whereas here, we adsorbed both, PLL and PLL- $g$-PMOXA from a physiological buffer solution $(10 \mathrm{mmol} / \mathrm{l}$ Hepes $+150 \mathrm{mmol} / \mathrm{l} \mathrm{NaCl})$ resulting in a higher lysine surface density for pure PLL.

[38] A. Kopf, J. Baschnagel, J. Wittmer, K. Binder, Macromolecules 1996, 29, 1433.
[39] R. Zajac, A. Chakrabarti, Phys. Rev. E 1994, 49, 3069.

[40] L. Feuz, F. A. M. Leermakers, M. Textor, O. Borisov, Macromolecules 2005, 38 , 8891.

[41] Y.-L. Ong, A. Razatos, G. Georgiou, M. M. Sharma, Langmuir 1999, 15, 2719. 